\title{
The Role of Surface Stress in the Morphology of Microbes
}

\author{
By ARTHUR L. KOCH, ${ }^{1 *}$ MICHAEL L. HIGGINS ${ }^{2}$ AND \\ RONALD J. DOYLE \\ ${ }^{1}$ Program on Microbiology, Department of Biology, Indiana University, Bloomington, \\ Indiana 47405, U.S.A. \\ ${ }^{2}$ Department of Microbiology and Immunology, Temple University School of Medicine, \\ Philadelphia, Pennsylvania 19140, U.S.A. \\ ${ }^{3}$ Department of Microbiology and Immunology, University of Louisville, Health Sciences \\ Center, Louisville, Kentucky 40292, U.S.A.
}

(Received 14 April 1981; revised 24 July 1981)

The shapes of many prokaryotes can be understood by the assumption that the cell wall expands in response to tension created by the osmotically derived hydrostatic pressure. Different organisms have different shapes because wall growth takes place in different regions. A previous paper (Koch et al., 1981a) considered the simplest case of prokaryotic growth, i.e. that of Streptococcus faecium. In the present paper, an elaboration of this theory is applied to two further cases - the more perfectly spherical cocci and the rod-shaped bacteria. These cases are more complex mathematically, because growth over a considerable fraction of the surface must be considered. Such diffuse growth cannot be treated analytically, but can be simulated on a computer or handled by geometric arguments.

The spherical form of the cocci may result from either diffuse growth over their entire external surface, or from zonal growth in which the addition of new material only occurs in the immediate vicinity of the splitting septum. In the zonal model, it must be assumed that the least amount of previously laid down septal peptidoglycan consistent with wall growth is reworked in the formation of the new external wall. For Gram-positive rods, where the body of the rod is truly cylindrical, three kinds of growth zones are required: (1) the inward edge of the ingrowing septum, (2) the junction of septum and nascent pole, and (3) the cylindrical walls. Two modes for cylindrical elongation are possible: (a) new wall is added in one or a few narrow annular zones, or (b) new wall material is added continuously all over the innermost surface and the outer layer is degraded. It is shown that the latter case applies to Bacillus subtilis.

Also summarized in this paper are results, developed in more detail elsewhere, concerning the morphology of fusiform bacteria, Gram-negative rods and the hyphal tips of fungi.

\section{INTRODUCTION}

In an earlier paper (Koch et al., 1981a), a model predicting the shape of the growing cell of Streptococcus faecium was presented. The model developed there was a specialized sub-model of a more general theory that may have validity in explaining the shapes of most prokaryotes. In this paper we present the application of this theory to other cases; we also present the formal treatment of growth over diffuse areas of the cell wall.

In its most general form, the theory asserts that the cell envelope enlarges during growth in special, limited regions of the stress-resisting peptidoglycan, which are different for organisms with different shapes. In these regions, peptidoglycan units or peptidoglycan oligomers are added to the existing structure without affecting the original strength. These new additions are 
not initially under the tension, due to hydrostatic stress, borne by the older wall. It is only after the new unit becomes covalently bonded at several sites to the wall that specific cleavages of the pre-existing wall take place. These cleavages occur in such a way that the newly inserted strands are no longer loosely held, but come under tension which pulls them into an extended configuration, brings them into the plane of tension, and thereby causes the enlargement of the wall. In regions of non-growing wall, it is assumed that further deformation due to the hydrostatic pressure is minimal.

The case of $S$. faecium appears to be especially simple. It is only necessary to assume that: (1) the zone of growth is very narrow and immediately adjacent to the ingrowing septum, (2) the septal material is already under tension as it becomes part of the external wall, (3) the septal wall is twice as thick as the immediately adjacent external wall at the instant of externalization, and (4) the hydrostatic pressure and wall growth activity is constant. The model derived from the foregoing assumptions was found capable of predicting the shapes of the growing organism, and also explained the occurrence of chains under growth conditions where the septal wall remains less than twice as thick as the external wall.

Among the more complex cases considered in the present paper is the growth of the nearly spherical cocci, such as staphylococci and gonococci. These organisms form a planar septum de novo across a diameter of the cell which then splits, expands, and eventually creates two new hemispheres as cell division ensues. Also considered are models for the elongation of cylindrical portions of rods for cases in which growth is narrowly zonal and in which it occurs diffusely. The zonal and diffuse growth models are also applied to the poles or caps of rod-shaped organisms. Finally, a range of shapes explainable by the surface stress model are summarized and evaluated.

\section{WALL GROWTH UNDER HYDROSTATIC PRESSURE}

In a closed vessel containing a fluid under pressure, forces are developed over the entire surface that are balanced by the tension developed in the wall. If that wall is inherently thin and flexible, then the forces normal to the wall must cancel; but tension remains in the plane of the surface. The magnitude of the tension depends on the size and shape as well as the internal hydrostatic pressure. To explain wall growth without cell rupture, it seems necessary that enlargement takes place by loosely attaching oligopeptidoglycan units or strands at several points with covalent bonds to the existing wall and then cleaving the older wall. Because tension would pull each new unit into the plane containing the tensile forces, the expanding wall would necessarily obey the same laws as soap bubbles do in forming the minimum surface consistent with other constraints. Such minimization, however, can lead to many of the varieties of known bacterial shapes.

The simplest form of a soap bubble is a sphere, since this is the shape that minimizes the surface for the contained volume. To a first approximation, one can explain the shape of the growing yeast bud by assuming that only in a single small region of the maternal cell's surface are the enzymic systems activated that allow wall growth over the entire bud surface. This corresponds to a constant surface tension, $T$. Akin to blowing a soap bubble, wall enlargement forced by the formation of additional incompressible protoplasm would lead to a spherical bud. This is an especially simple example because there are only two constraints specified by the biological system. They are: (1) at bud initiation, wall growth occurs more readily in a single small compact region of the surface of the maternal yeast cell, and (2) growth occurs uniformly over the entire surface of the wall of the bud. In other words, yeast-type growth results when there is a localized decrease in $T$ at one spot on the walls and $T$ then remains uniform over the surface of the growing bud; as the bud grows, however, $T$ progressively increases and the new cell may, in turn, also bear buds.

The cases of bacterial growth that are considered here have more complicated constraints. The method developed earlier (Koch et al., 1981a) applies if growth takes place only in a 
narrow, cylindrically symmetric region of the cell, and would not apply if growth is diffuse so that a larger region is subject to shape adjustment as growth ensues. Therefore, a more complex mathematical approach must be made.

\section{UNDULOIDS}

Young (1805) and Laplace (1806) discovered a relationship between the pressure difference, $P$, across a tensile membrane and any two perpendicular radii of curvature:

$$
P=T\left(1 / R_{1}+1 / R_{2}\right)
$$

In this formula, $T$ is the surface tension and $R_{1}$ and $R_{2}$ are usually chosen as the maximum and minimum radii of curvature. The equation applies at any point on the surface. The problem sufficiently general for our needs is that of determining the shape of a cylindrically symmetric surface bearing a pressure difference and constrained by two rigid coaxial circles of radius $a$ and $b$ separated by a distance $L$, as shown in Fig. 1 . One possible surface is shown; this is the catenoid and applies for the special case where the pressure difference across the membrane is zero. Other shapes, more bowed out or in, would be produced if the internal pressure were higher or lower than the external pressure. In the biological applications presented below, the symbol $a$ will be used for the maximum radius of the cell, i.e. as the radius of the cylindrical part or the base of the pole or cap of the cell.

Unfortunately, there is no general mathematical solution to the differential equations for this case. Delaunay (1841) and Maxwell (1849) gave a formulation that allows the generation of all the possible curves. Their construction is rather like that for a cycloid. The curves are generated by rolling any conic section along a straight line. Then the path of the focus of the conic section when rotated around the line leads to the surfaces of interest (see Maxwell, 1927; Thompson, 1942). The curves that can be generated if the conic section is an ellipse are called 'unduloids'. For a few of the limiting cases, analytical solutions are available. For example, if the conic section is a circle, then its centre while rolling along a straight line generates a parallel line. The subsequent rotation of this generated line about the original line produces a cylindrical surface. This case is relevant to the generation of the side walls of bacterial rods. This solution applies if: (1) the two constraining radii, $a$ and $b$, are equal and equal to $R_{1},(2) R_{2}$ is infinite, and (3) the pressure $P$ is equal to $T / a$. A larger internal pressure will lead to a barrel-shaped structure and a smaller pressure to an inward curvature. However, even when $P=T / a$, if the distance is greater than the circumference of the cylinder, the system is unstable and will spontaneously decompose into two or more spherical surfaces (see Maxwell, 1927; Thompson, 1942; Isenberg, 1978). This phenomenon is the basis for the

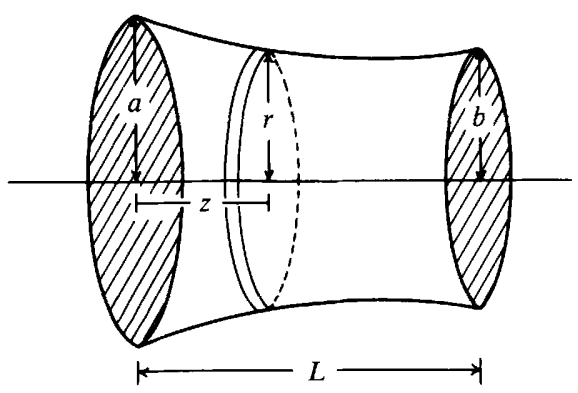

Fig. 1. The unduloid. An example of an unduloid is shown. The surface is constrained to span the region between two coaxial circles of radius $a$ and $b$ which are separated by a distance $L$. The surface shown corresponds to no pressure difference between the inside and the outside. Other unduloids with the same boundary values, but more convex or concave, would be produced if the internal pressure were higher or lower than the external pressure. 


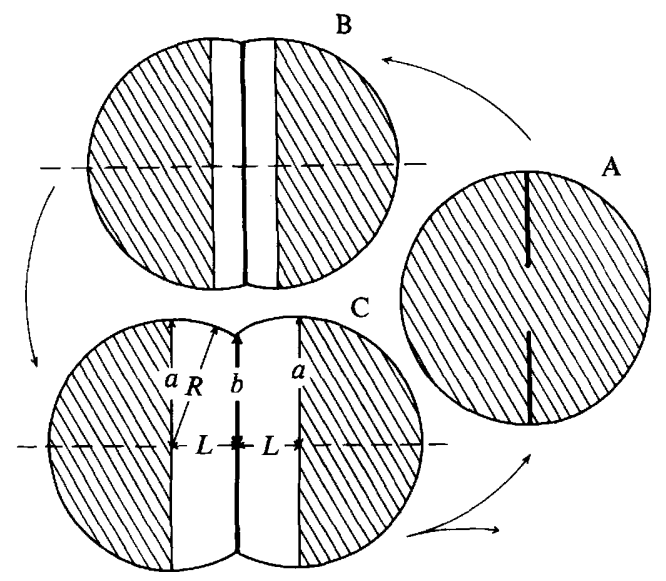

Fig. 2. Idealized model for diffuse wall growth of spherical staphylococci or gonococci. In A, the spherical cell, newly produced by cell division is shown. In B, the septum has completed and partially split. In $\mathrm{C}$, this process has continued further. The unshaded areas represent regions of new wall growth. In this diffuse model, new wall is added at least over the entire new poles. All surfaces are spherical with radius $R$. Growth of wall in the new poles maintains the spherical curvature because of special relationships between $a, b, L$ and $P / T$ (see text).

formation of droplets from a jet of fluid. Its relevance to bacterial cell division will be discussed below.

The spherical surface segment (or a complete spherical shell) is the second special case of an unduloid for which an exact formulation can be written. Figure 2 shows an intermediate in the idealized cell growth cycle of a staphylococcus or gonococcus. The unshaded areas represent regions of new wall growth. Either half is equivalent to Fig. 1 in that it represents the minimal surface between two coaxial rings, but the spherical nature leads to the restriction that $r=a$ and $r^{2}=b^{2}+L^{2}$. In this special case, the two perpendicular radii of curvature at any point on the surface will be equal and equivalent to $r$. It follows that the value of $T\left(1 / R_{1}\right.$ $+1 / R_{2}$ ) will be constant all over the spherical surfaces. This means that we have guessed, and shown, that a sphere can be a correct solution. However, for this special case, equation (1) requires that (in addition to $r=a$ and $\left.r^{2}=b^{2}+L^{2}\right) P, T$ and $r$ must satisfy the relationship:

$$
P=2 T / r
$$

This relationship provides an explanation for the growth of nearly spherical cocci (see below).

The third case for which an explicit mathematical expression can be given is that of the catenary of revolution (the catenoid). This is the surface depicted in Fig. 1, which results from rolling a parabola along a line, and applies if there is no pressure differential across the surface (see Isenberg, 1978). For other reasons, the catenoid may describe the shape of the pole (A. L. Koch, unpublished results).

\section{COMPUTER GENERATION OF UNDULOID SHAPES}

To calculate the profile of unduloids with a modern computer we could have carried out Maxwell's geometric procedure analytically. It appeared more convenient, and in fact more general, to go back to the derivation of equation (1) and re-express this in a form expedient for numerical integration. This can be done readily by calculating the free energy, $G$, inherent in the surface and the work, $W$, of forming the volume against the pressure, $P$. We then minimize the sum of these two quantities using the methods of the calculus of variation. The free energy term is: 


$$
G=\int T \mathrm{~d} A=2 \pi T \int_{0}^{L} r\left(1+S^{2}\right)^{1 / 2} \mathrm{~d} z
$$

In this expression, $r$ is the radius from the axis of cylindrical symmetry, $z$ is the axial distance, and $S$ is the slope. The work term is:

$$
W=\int P \mathrm{~d} v=\int_{0}^{L} P \pi r^{2} \mathrm{~d} z
$$

Therefore the functional to be minimized is:

$$
F=\int_{0}^{L}\left\{2 \pi \operatorname{Tr}\left(1+S^{2}\right)^{1 / 2}-\pi \operatorname{Pr}^{2}\right\} \mathrm{d} z=\int_{0}^{L} I \mathrm{~d} z
$$

Consequently, the Euler-Lagrange equation is:

$$
2 \pi T\left(1+S^{2}\right)^{1 / 2}+2 \pi \operatorname{Pr}-\mathrm{d}\left\{2 \pi \operatorname{Tr} S /\left(1+S^{2}\right)^{1 / 2}\right\} / \mathrm{d} z=0
$$

Since the integrand in equation (5), $I$, is independent of the axial distance, another form equivalent to this is:

$$
I-S \delta I / \delta S=\text { constant }=C
$$

After substituting equation (5) into equation (7), rearrangement yields:

or

$$
\begin{gathered}
2 \operatorname{Tr} /\left(1+S^{2}\right)^{1 / 2}=C+P r^{2} \\
S=\left[\left\{2 \operatorname{Tr} /\left(C+P r^{2}\right)\right\}^{2}-1\right]^{1 / 2}
\end{gathered}
$$

In theory, the initial values of $r$ and $S$, which will be designated $r_{0}$ and $S_{0}$, can be substituted into equation (8). This defines $C$, which then could be used in equation (9) to calculate the slope at any point of the profile of an unduloid that passes through the radius $r_{0}$ with a slope $S_{0}$. In practice, this form is inappropriate unless the computer carries very many decimal places. Alternatively, equation (8), written for the initial values, can be substituted into equation (9) so as to eliminate $C$. The results can then be rearranged in such a way that subtraction of almost identical quantities can be done with little rounding error. This rearranged equation, used by our computer program, is:

$$
S=\left\{M(M-2 N) / N^{2}\right\}^{1 / 2}
$$

where

$$
M=(P / T)\left(r_{0}-r-\Delta\right)\left(r_{0}+r\right)+\left\{2 r-2 r_{0} /\left(1+S_{o}^{2}\right)^{1 / 2}+2 \Delta\right\}
$$

and

$$
N=(P / T)\left(r_{0}-r-\Delta\right)\left(r_{0}+r\right)-2 r_{0} /\left(1+S_{o}^{2}\right)^{1 / 2}
$$

In our program, double precision ( 24 decimal places) is used in sensitive parts of the computation. In these expressions $\Delta$ is a residue of the second 12 decimal places involved in the computation and would not apply and should be deleted if the computer uses a different mode of carrying out high precision arithmetic.

The numerical integrations reported here have been carried out in a simple 'brute force' way. The computer calculated $r$ for a small $\mathrm{d} z$ step from the previous $r$ and $S$ values and then computed a new $S$. It repeated this cycle tens of thousands of times to calculate a branch of each curve. The program has the facility to adjust the size of the steps under specified conditions and to terminate when the slope becomes zero or imaginary. While more efficient computation could be carried out using big computers, the minicomputer yields accurate profits although lengthy computations are needed. The program in a form suitable for a Wang 720 is available from the authors. The program also yields as output the surface area and contained volume. The validity of the program was tested by choosing parameters that should yield spheres, cylinders and catenoids. The agreement was excellent. 


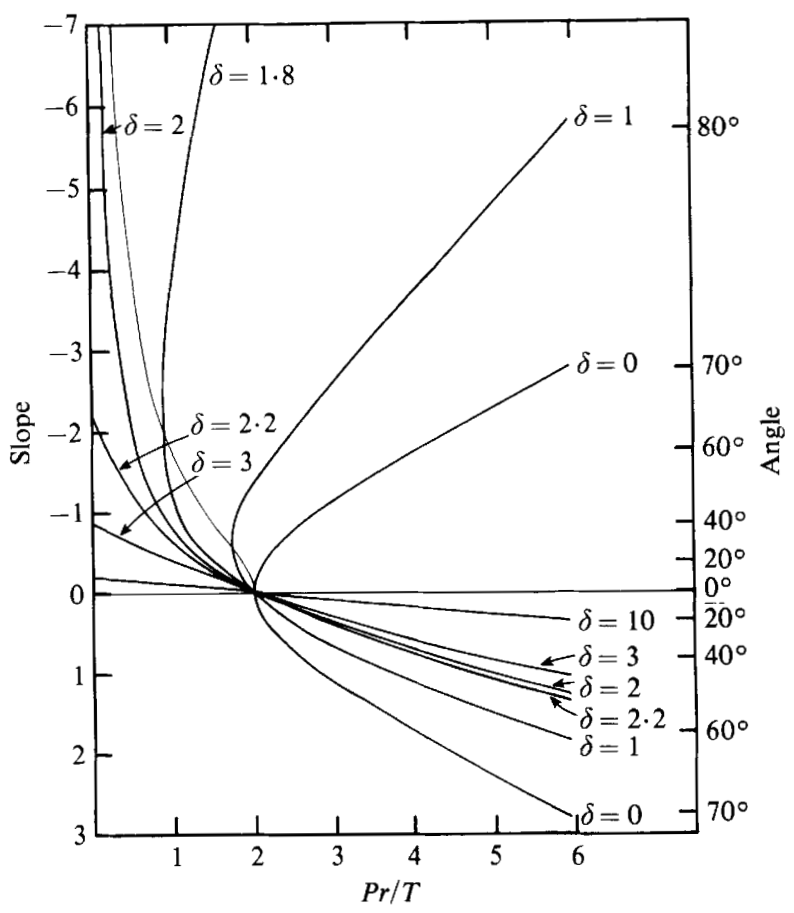

Fig. 3. Relationship between the slope $(S)$ of the zonal growth area and $\mathrm{Pr} / T$. Each curve corresponds to the indicated value of $\delta$. These are plotted against the slope on the left-hand ordinate and against the angle of new growth on the right-hand ordinate. Note that all curves go through the point corresponding to $S=0, \operatorname{Pr} / T=2$. Also, only for $\delta \geqslant 2$ do the curves reach $\operatorname{Pr} / T=0$, corresponding to the possibility of cell division. The thin, unlabelled curve corresponds to the least values of $\operatorname{Pr} / T$ for values of $\delta<2$. Growth along this path corresponds to 'i-driven growth' (see text).

\section{ZONAL GROWTH}

Koch et al. (1981a) developed a general formula for the wall growth of narrow zones that are subject to internal hydrostatic pressure. The algebraic expression that resulted from this analysis is plotted in Fig. 3. This plot differs from that published previously (Fig. 5 of Koch et $a l ., 1981 a$ ) in that a larger range of values of $\delta$ is shown. The definition of $\delta$ is the ratio of the thickness of the septum relative to the external wall. Having no septum at all corresponds to $\delta$ $=0$. This figure will be used below to consider cylindrical elongation when $\operatorname{Pr} / T=2$ and the development of fusiform bacteria when $\delta>2$. When $\delta=2$ and $\operatorname{Pr} / T$ is less than 2, pole development takes place. The shape of the pole is determined by the ratio of pole height to width. The observed shape of $S$. faecium was found to be accurately fitted to the formula derived for this case by a suitable choice of a single parameter, $P / T$. The shape of the resultant pole cannot be spherical no matter what value is chosen for $P / T$.

\section{FORMATION OF CHAINS}

Koch et al. (1981a) showed that if the septal thickness is twice that of the external wall and $\mathrm{Pa} / \mathrm{T}$ is less than 2, then zonal growth leads to pole development and finally results in binary fission. However, when $\delta$, the ratio of septal wall thickness to the thickness of the external wall, remains less than 2 , pole development cannot be completed because eventually $\operatorname{Pr} / T$ decreases so that the slope of the growth zone becomes imaginary and further constriction cannot take place (see Fig. 3). For example, if $\delta$ remains constant at 1 or $1 \cdot 8$, growth could 
take place along one of the dashed lines shown in Fig. 4(a). As pole growth proceeds, $r$ becomes progressively narrower and constriction would occur more rapidly. When growth proceeds to intersection with the continuous line (which is the locus of points at the minimum values of $S$ versus $\operatorname{Pr} / T$ curves), further enlargement of the growth zone cannot take place. At this point, there is no slope that the new wall can have that will accommodate the newly produced cytoplasm inside the new surface area and at the same time conserve the pressure-volume work in the expansion of the wall. Cessation of wall expansion leads to a temporary pressure increase, which we suggest triggers the development of new growth zones that, in turn, lower the pressure. The result is that chains of organisms will be produced. The chains are linear in the case of streptococci, in which the planes of successive divisions are parallel to each other. In the case of staphylococci, the successive divisions are nearly perpendicular to each other and grape-like clusters of cells result. This kind of process also yields grape-like clusters and groupings such as those exhibited by Sarcina and Lampropedia.

\section{GROWTH OF SPHERICAL COCCI}

Staphylococci and gonococci grow in a manner quite distinct from the streptococci as typified by Streptococcus faecium. Their shape is much closer to that of a sphere than is $S$. faecium and the cross-wall or septum develops as an annular ring that, when completed, may become a disc of nearly the same radius as the organism (Giesbrecht et al., 1976). The cell thus forms an equatorial septum through the cytoplasm. The cell division process is shown in an idealized way in Fig. 2. When completed or nearly completed, the disc starts to split. As the volume of the cell increases, the septum splits more and the newly split part bulges out so that the new wall becomes part of a spherical surface continuous with the old pole of the cell. Thus, one sphere becomes two spheres while the radius $a$ stays constant. Two possible mechanisms will generate such spherical growth within the general context of the proposed model. Each will be presented and its further implications considered.

\section{Zonal $i$-driven growth}

Also shown in Fig. 3, as a thin continuous line, is the locus of the smallest real value of $\mathrm{Pr} / \mathrm{T}$ of the different curves for fixed values of $\delta$. This line determines the development of a pole for the case in which the septal contribution at all stages of pole development is the least possible that allows pole growth. Such a mode of pole formation has been designated 'i-driven growth' (Koch et al., 1981 a) and is a possible explanation for the formation of spherical poles as well as of certain types of non-spherical poles. When pole formation must halt because the values of $\mathrm{Pr} / \mathrm{T}$ and $\delta$ correspond to an imaginary slope, there is another possible growth regulatory mechanism: $\delta$ may be increased just enough so that $S$ is real once again. It is this alternative that generates hemispherical poles when an additional condition is met. The second condition needed for a spherical shape is that the parameter $\delta$ must be zero when splitting of the transverse septum is initiated. In this case, the trajectory of the development of the pole is shown by the continuous curve in Fig. $4(a)$ as a plot of $S$ versus $\operatorname{Pr} / T$. As pole formation takes place, $\operatorname{Pr} / T$ goes from 2 to 0 and simultaneously $\delta$ goes from 0 to 2 while $S$ goes from 0 (or $0^{\circ}$ ) to $-\infty$ (or $90^{\circ}$ ). The shape of the completed pole is shown in Fig. $4(b)$ by the curved marked $\delta=0$.

Also shown in Fig. 4(a) is the course of the increase in slope as the pole develops for cases where initially $\delta=1$ or 1.8 and remains at these values until further growth is no longer possible, but then $\delta$ increases according to the $\mathrm{i}$-driven growth mode which leads to the completion of cell division. The shape of the completed poles for the three cases shown in Fig. $4(a)$ is shown in Fig. $4(b)$. The arrows indicate the points in the development of the pole where growth becomes $\mathrm{i}$-driven. A prediction of this model for hemispherical poles is that very little material previously present as septum should appear in the external wall early in the 

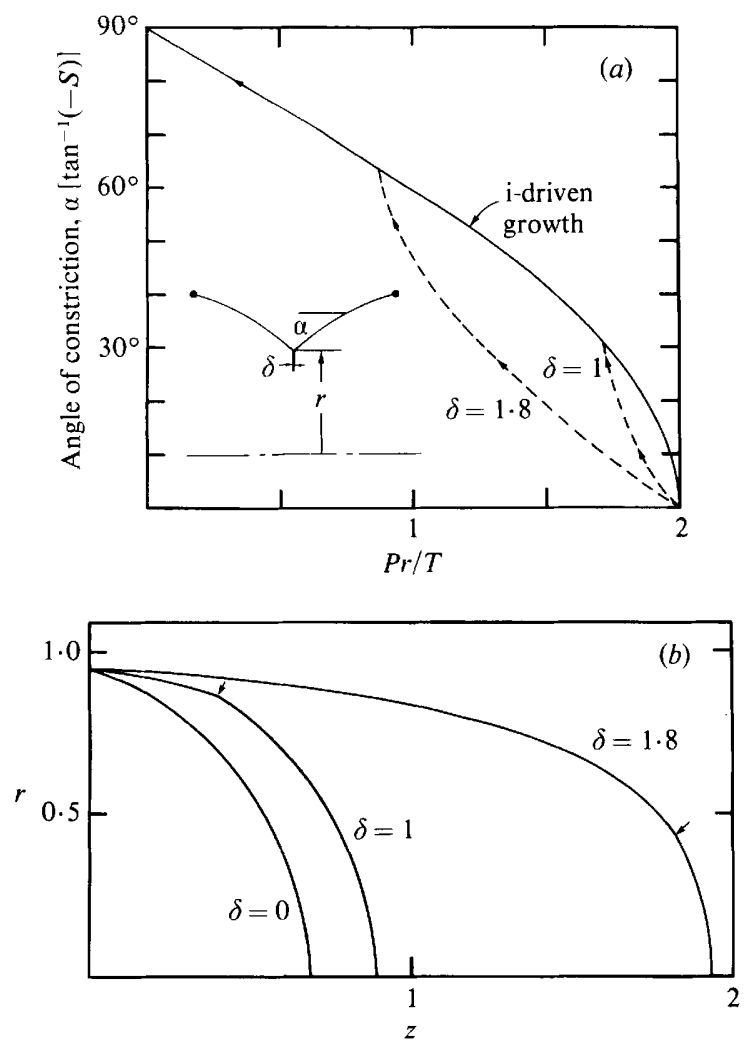

Fig. 4. (a) Development of poles completed by i-driven growth. The inward growth of the pole is shown for three cases where the initial values of $\delta$ are 0,1 and 1.8. The last two are indicated by the dashed lines. A continuous line is used for the former case and for the portion of the curve for the other two cases when growth becomes i-driven because the pole has progressed to the point where the slope becomes imaginary. The plot shows the angle of growth (see inset) versus the value of $\mathrm{Pr} / \mathrm{T}$.

(b) The one-quarter profile of the three cases in $(a)$. Arrows indicate the points in pole development when growth becomes i-driven. Note that the full i-driven case results in a circular profile. $P a / T$ has been set to 1 for this illustrative example.

life of the nascent pole. Conversely, the septal material should form the bulk of the external material in the last moments before cell division for both i-driven and zonal models.

\section{Formation of spherical cells via diffuse growth}

In contrast to the zonal model, diffuse growth occurs when new peptidoglycan is added to the nascent pole, not just in the region of its junction with the septum, but all over the nascent pole (or indeed all over the wall of the entire cell). When the entire wall is capable of enlargement, it should have many of the properties of ordinary soap bubbles. However, simply having the properties of a soap bubble will not account for the growth of the nearly spherical cocci. This can be demonstrated by blowing two identical spherical bubbles and allowing them to fuse to form a doublet. A planar septum forms between the two spherical surfaces and the angle between the spherical surface and the planar septum is exactly $120^{\circ}$. If two larger bubbles were fused, the resultant complex would be similar in shape - merely bigger. Consequently, a strict soap bubble model for growth would never lead to cell division, rather to bigger diplococci (if a transverse septum were formed). A second point of difference between an ordinary soap bubble and the actual bacteria is that if the two fused soap bubbles were enlarged the pressure within each would decrease according to equation (2). On the 
other hand, the bacteria (as judged from their fixed radii) maintain their internal pressure. This suggests an analogy to the $S$. faecium case, in that the pressure is maintained constant by the cell's adjustment of the rate of wall growth in a homeostatic response to internal pressure.

For the moment, assume that the unsplit septum in Fig. 2 is rigid and that the old pole retains a fixed shape as growth of the new poles continues. As shown above, among the possible unduloids which can satisfy these boundary conditions, one unduloid has a spherical shape. However, growth in a spherical form can only happen if the septum splits and the nascent pole forms in a special coordinate way with the protoplasmic growth which forces enlargement of the wall to take place: only if $b$ and $L$ change so that $a^{2}=b^{2}+L^{2}$ can equation (1) be satisfied at all points of the nascent pole. Thus, a spherical shape is consistent with constant values of $P$ and $T$ over the entire nascent growing pole. Now we can relax the assumption that the old pole and the septum are rigid. If the enzymes involved in wall enlargement were active over the entire area of the cell surface, then with constant $P$ and $T$ only this kind of extension of a spherical surface with the particular radius, $a=2 T / P$, would be energetically possible. Thus, the model for diffuse growth predicts that throughout the growth cycle new material would be added all over the surface. Consequently, the relative distribution of newly inserted peptidoglycan would be crucial in choosing between the diffuse model and the i-driven zonal model for hemispherical poles. Obviously, another critical test of this diffuse model is the detailed localization of the family of enzymes involved in wall enlargement. This includes the enzymes involved in formation of cross-bridges and, especially, the enzymes involved in the splitting of the septum and the lytic actions that permit expansion of the wall.

The argument developed in this section can be turned around. The only conic section that can be rolled along a line and have its focus periodically touch the line is an ellipse with an infinite eccentricity. Such a degenerate ellipse is a straight line segment. When 'rolled', its focus generates a path consisting of a row of contiguous closed hemispheres. Consequently, the only shape that a cell can have which is uniformly capable of wall growth all over its entire surface and depends on surface tension-like properties to lead to the cell division is that of a sphere. If $T$ varies over the surface in special ways (see Koch, 1982b), surface tension-like forces can lead to cell division with non-spherical poles.

\section{GROWTH OF CYLINDRICAL REGIONS OF BACTERIA}

Like the formation of poles, the elongation of the cylindrical regions of rod-shaped bacteria is a process that also can be understood in terms of both zonal and diffuse growth.

Although the evidence is presently not fully compelling (Koch, 1982b), it is probable that the walls of Gram-negative enteric organisms are laid down by a diffuse process where $T$ is varied in a particular way. However, for the case of the typical Gram-positive organism, Bacillus subtilis, there is good evidence that growth takes place diffusely over the entire cylindrical surface, but only in a narrow zone at the splitting system for the development of poles.

Evidence for diffuse growth of the entire cylindrical regions of $B$. subtilis comes from the experimental observations of Fan et al. (1975), Pooley (1976a,b), Archibald (1976) and Archibald \& Coapes (1976). These workers, by biochemical and electron microscopic studies, found that the bulk of the peptidoglycan enters the wall fraction on the inner membrane and subsequently moves through the wall. In wild-type organisms the peptidoglycan is finally sloughed off into the external medium. These important experiments and the surface stress theory led to the version of the general model appropriate for growth of Gram-positive, cylindrical cells. The model was introduced previously (Koch et al., 1981 $a, b$ ) and is shown in more detail in Fig. 5. New oligopeptide units are linked to form a loose fabric between the cytoplasmic membrane and the older wall material. If a cohort of peptidoglycan 


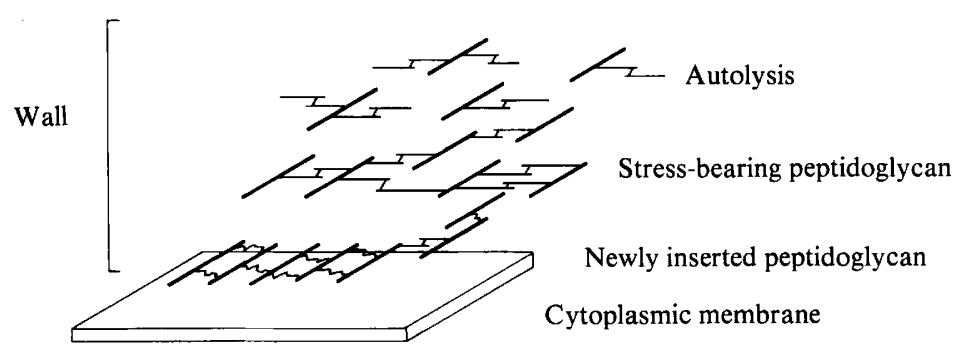

Fig. 5. Elongation of the cylindrical surface of Gram-positive rods. Peptidoglycan is laid down and cross-linked while not under tension on the inner face of the stress-bearing peptidoglycan. As further layers are added internally and as other layers are autolysed, stress develops and causes the elongation and expansion of a layer (see text). Note that it is possible to make a similar model where the glycan chains grow normal to the cell membrane.

units are followed in time, the model proposes that they are first linked to each other (and in some degree to the older wall). When they, in turn, are displaced outward and come to bear the stress, the network expands, permitting growth. During this second phase, no covalent bonds form. Later, as the cohort moves further peripherally, bonds are cleaved permitting a more internal layer to bear the stress and expand. In mutants deficient in wall-degrading enzymes, at least some bonds must be cleaved, even though the old peptidoglycan may remain attached to the wall.

While theoretically a diffuse mechanism can function for side wall elongation and for hemispherical pole formation, the two processes cannot occur simultaneously in the same cell with the same values of $P$ and $T$ applying to each. In such a case, the cell would become spherical. Critical experimental evidence that the pole wall is metabolically different from the cylinder wall has come from experiments in which chemostat cultures of $B$. subtilis were shifted from potassium to phosphate limitation (Archibald \& Coapes, 1976; Anderson et al., 1978; Sturman \& Archibald, 1978). Only with adequate phosphate did the cells form teichoic acid in the wall. The sites of wall growth and turnover were identified by the ability of the wall to bind the bacteriophage SP50. A short growth period in one kind of limitation followed by a long chase in the other limitation showed that poles, once formed, were stable, and that only the side walls participated in inside-to-out diffuse growth. This model also explains the recent finding of Doyle et al. (1981) that wall turnover consists of two phases - a rapid, major one and a much slower, minor component. From quantitative turnover studies and fluorescence microscopy, they attribute greater metabolic stability to the poles and septa than to the side walls. Both types of experiments show that it is the poles that are conserved and not a larger unit, sometimes designated as the 'unit cell', consisting of the pole plus one half of the cylinder length of a new-born cell. Further discussion of this point is given elsewhere (Koch et al., $1981 b)$.

\section{Zonal elongation}

Although a diffuse model applies to $B$. subtilis, a zonal model could function for cylindrical extension in other cases and, indeed, has been assumed by most workers for all cases. In the mathematical formulation for zonal growth developed previously, an equation for $S$ was developed which depended on $\mathrm{Pr} / \mathrm{T}$ and $\delta$. Here, we are interested in the case where there is no septum and $\delta=0$. The formula for the slope when $\delta=0$ is:

$$
S= \pm\left\{(\operatorname{Pr} / 2 T)^{2}-1\right\}^{1 / 2}
$$

It can be seen from this equation that only when $P r / T$ is 2 will the slope be zero. Elongation of a rod of radius $r=a$ will occur (if $P / T$ is of the proper value, i.e. equal to $2 / a$ ) in such a way that the new region will neither tend to constrict nor expand the radius of the cell. 
When the quantity $\operatorname{Pr} / T$ is less than $2, S$ is imaginary and wall growth cannot take place. If anabolism and transport of solutes increase the hydrostatic pressure, or if the function of wall-synthesizing enzymes becomes altered in such a way as to lower $T$ so that $\mathrm{Pr} / T$ increases to at least 2, wall growth may commence. If $\mathrm{Pr} / \mathrm{T}$ is greater than 2 , zonal wall growth can take place, but there are two possible slopes for the new wall depending on whether the plus or minus sign in equation (13) is appropriate to the biological conditions.

If the sign is positive, the cell, upon elongation, will become wider. The chance fluctuation to a larger diameter could be catastrophic if $P$ and $T$ for the growth zone were constant, since further growth would lead to further dilation. This circumstance tends to make zonal cylindrical growth unstable unless the cell has some way of providing circumferential adjustment if it becomes too wide. It is difficult to imagine how such a mechanism could sense the diameter of the cell or how it would alter $P$ or $T$.

If the slope is negative, then the growth zone will have a negative slope and tend to constrict. If $P$ and $T$ remain constant as the growth zone continues to form new wall, elongation leads to further, but less marked, constriction. As $r$ progressively decreases, $P r / T$ approaches 2 . Thereafter, the radius of the growing zone remains constant. If initially $\operatorname{Pr} / T$ is very large, the decrease will approximate a negative exponential. The shift of an enteric organism to a poorer environment and its concomitant decrease in $r$ can be explained in this way.

The positive branch of the curve could be used to account for formation of 'fatter', wider cells when Gram-negative organisms are shifted up into a richer medium. As growth continues in the new medium, the cell becomes wider, and eventually a new state of balanced growth with a larger, stable cylindrical radius may be achieved. The widening could be because $P$ increases or $T$ decreases as the result of the shift. However, some other regulatory mechanism must be involved to prevent the widening from continuing unchecked.

\section{Diffuse elongation}

While it is readily possible to blow a cylindrical soap bubble, it is not at all obvious what the conditions could be, if any, such that further growth would lead to elongation and not to conversion of the cylindrical shape towards a spherical shape. The theoretical conditions leading to diffuse elongation were studied with the computer program described above. First, the well-known instability of long, cylindrical surfaces was used to test the precision of the numerical methods employed here in a rather stringent way. Instability was only found when $L$ approached $2 \pi a$ closely. The computer then calculated the profile for a barrelled-out cylinder with a pressure $1 \%$ in excess of that needed to maintain a perfect regular cylinder. For cylinders shorter than $2 \pi a$, it was found that this shape was well approximated by a sine function. Assuming a sine wave shape, the energetics of new synthesis of wall accommodating the new synthesis of protoplasm could be compared analytically for two different models of diffuse growth: (1) growth resulting in bulging, and (2) growth leading to elongation (Fig. 6). For a fixed, small volume increment, the sine wave approximation showed that the choice in terms of minimizing wall growth is in favour of bulging. However, the difference in area for the two growth modes is small. The ratio of the increase in surface area for elongation, $\Delta A_{E}$, to that for bulging, $\Delta A_{\mathrm{B}}$, is:

$$
\Delta A_{\mathrm{E}} / \Delta A_{\mathrm{B}}=1+4 \Delta r / \pi r
$$

where $\Delta r$ is the increase in radius in the case of bulging. Thus, $\Delta A_{\mathrm{E}} / \Delta A_{\mathrm{B}}$ is always greater than 1 but approaches 1 as smaller increments of growth are considered and therefore as $\Delta r$ approaches zero.

The fact that bulging and cylindrical extension are of similar energy means that other very small forces could favour elongation instead of bulging. If growth of a Gram-positive rod is diffuse, with each inner layer expanding as it moves outward and comes under stress as 




Fig. 6. Diffuse elongation of rod-shaped organisms with fixed radii at the ends. Two possible modes of volume expansion of a cylinder are shown.

proposed above, the shape of the older, previously stress-bearing, wall could bias the course of expansion.

But even if a bulge forms, it would only be temporary. Equation (1) requires that the bulged shape could only be achieved if the pressure increased above the value that makes $P a / T=1$. Of course, protoplasmic growth tends to increase the pressure. However, if wall growth continued as long as the pressure was greater than a critical value, expansion would continue until the pressure returned to its original value and, hence, to a cylindrical shape. The deviation from a perfect cylinder for certain rod-shaped organisms, and certainly for $B$. subtilis, is small and therefore the rectification of any temporary or intermediate bulging must occur quickly and effectively; continued growth returns the radius to a value given by $a=$ $2 T / P$.

The implications of equation (1) for diffuse cylindrical elongation are far-reaching. If the hydrostatic pressure is maintained by cell mechanisms which regulate the rate of wall growth, although not its geometric location, then elongation instead of bulging would occur if the poles were rigid. To show this, it is only necessary to compare equation (1) for the case when $R_{1}=a$ and $R_{2}=\infty$, with the same equation for the case when $R_{2}$ is less than infinity. If $P$ and $T$ are the same for the two cases, then $R_{1}$ for the second case must be greater than $a$. This means that for the cylindrical surface to bulge, the radius at the ends must increase, making expansion of the poles a necessary accompaniment to the bulging of a previously cylindrical region. This leads to the important conclusion that cylinder elongation by diffuse side wall growth can take place only if the poles are rigid and constant pressure is maintained.

The zonal mechanism for cylinder elongation would also work if, instead of a single growth zone, there were multiple, but well separated, growth zones. As long as the relationship $\mathrm{Pa} / \mathrm{T}$ $=2$ applied to each zone, cylindrical elongation at constant diameter would occur. There is a contradiction, however, for if there were a very large number of growth zones, the diffuse case would be approached and, as described above, $\mathrm{Pa} / \mathrm{T}$ should be 1 in order for cylindrical growth to occur. Consequently, the two kinds of models are mutually incompatible. It can be questioned how narrow a growth zone must be in order to be described by the equation for the zonal model we have developed previously. The unduloid computer program described above can be used to answer this question. Figure 7 shows, on a double logarithmic plot, the degree of bulging that would be produced with different widths of growth zones at different pressures. Clearly, a narrower zone resists the tendency to bulge much more effectively than do wider zones.

There is a limit to the extent of cylindrical elongation due to diffuse growth: a cylinder is not necessarily the only possible unduloid surface connecting two rings of the same diameter even when $\mathrm{Pa} / \mathrm{T}$ is 1 . As mentioned above, a cylindrical soap bubble becomes unstable and leads to flickering structures that alternately bulge at one end and then the other end as $L$ 


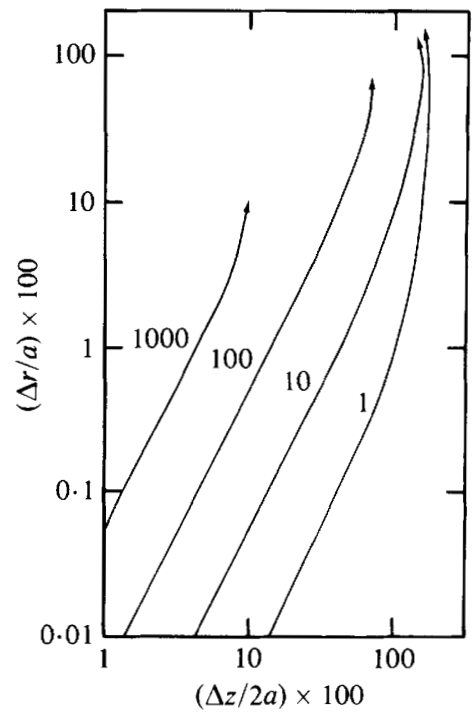

Fig. 7. Diffuse growth in zones of different width. The figure shows the bulging produced when zonal growth takes place on a cylindrical annulus of varying proportions and $P / T$ has values that cause bulging. The numbers on the graphs are the percentages by which $P / T$ exceeds the $P / T=1 / a$ values. $\Delta z$ is the width of the growth zone. The arrowheads indicate the greatest increase in radius at a given pressure obtainable with any width growth zone.

approaches $2 \pi a$. Eventually, the cylinder splits into two unequal spherical shapes. As long as $L$ is less than $2 \pi a$, this does not happen and a cylinder of uniform diameter is the only mathematical and physical solution.

As noted above, uniform diffuse growth cannot lead to division of one object into two objects without additional biological mechanisms unless very special conditions are met. The special case is if a cylindrical bubble becomes too long, then it will spontaneously separate into two. This process does not, however, lead to equipartition of the volume. Rather, a bulge forms near one end and a constricted zone appears near the other end. Eventually, the first region broadens and the other region constricts further leading to separation. This cannot be a model for cell division, not only because of the unequalness of the division, but because it requires a shrinkage of the cell wall in the region of constriction as well as an enlargement elsewhere.

\section{GROWTH OF POLES IN ROD-SHAPED BACTERIA}

\section{Zonal dome, diffuse growth and Cassinian models}

Since $P a / T$ is 2 and $\delta$ is 0 during cylindrical zonal extension of rod-shaped organisms, the simplest model of pole development is that the constriction process is started by building peptidoglycan inward to form a septum, so that $\mathrm{Pa} / \mathrm{T}$ becomes less than 2 and $\delta$ greater than 0 . This would require an additional biological mechanism responsive to the degree of growth of the cell; it is usually assumed that this is mediated via a stage of replication of the chromosome. As shown previously (Koch et al., 1981a), when internally deposited peptidoglycan is available for externalization, the constriction process is energetically favoured. Mathematically, if $\delta$ is greater than zero and $\mathrm{Pa} / T$ is less than 2 , then pole formation will commence and division will ensue as $\operatorname{Pr} / T$ decreases toward 0 and $\delta$ reaches 2 .

The shape of the poles so created can be calculated. For the particular case where $\delta$ is 2 throughout the development of a pole, we have shown that:

$$
z=(2 T / P) \ln \left[\left\{1-(P r / 2 T)^{2}\right\} /\left\{1-(P a / 2 T)^{2}\right\}\right]
$$






Fig. 8. Shapes of bacterial cells of zonal dome, diffuse (spherical) and Cassinian models. Each curve represents one-quarter of the profile of a newly divided cell. The values of $P / T$ employed are shown on each curve. The continuous lines show cell shapes for the zonal growth model where $\delta=2$ throughout the development of the pole. The dash-dotted lines are for the case of diffuse growth, and are portions of circles. The dashed lines are for the Cassinian model. Values of $n$ were chosen so that $z_{\max }$ would be the same as for those curves plotted for the zonal model: $n=6.71,3.01,1.356$ and 0.9387 correspond to $P / T=1 \cdot 8,1 \cdot 6,1$ and 0.5 , respectively.

where $z$ is the axial height at which the radius is $r$, and $a$ is the radius at the base of the pole where $z=0$. Equation (15) is equivalent to equations (4) and (5) of Koch et al. (1981a). We designate poles formed according to equation (15) as having a 'zonal dome' shape. Figure 8 shows profiles of zonal domes calculated from equation (15) for the radius at the base fixed, but differing in the assumed value of $\mathrm{P} / \mathrm{T}$ (continuous lines). Also shown are curves (Fig. 8, dash-dotted lines) that would apply if growth took place all over the pole surface during its development. These are, of course, segments of spherical surfaces. Obviously no choice of $P / T$ for this case of uniform diffuse growth can yield a maximum value of $z$ greater than $a$.

While we have not yet done extensive fitting of these models to electron microscopic profiles of cells, measurements of the contours of a variety of bacteria have been carefully made and fitted by E. Oldmixon (unpublished results). In his work, measurements from electron micrographs of individual cells were fitted by non-linear least square treatment to various theoretical shapes. Oldmixon was able to rule out ellipsoidal shapes (including spherical) for the cell pole of various rod-shaped organisms. However, he found very close agreement with an obscure mathematical form, the Cassinian equation:

$$
z=1 / n\left[a^{2}-r^{2}+2\left\{a^{2}\left(a^{2}-r^{2}\right)\right\}^{1 / 2}\right]^{1 / 2}
$$

(He chose this form for study because of superior mechanical properties of boilers with ends made of this shape.) The Cassinian could be fitted very precisely to measurements on the poles of a variety of rod-shaped bacteria. Only the 'affine' constant, $n$, which determines the relative proportions was estimated from the measurements. The maximum radius of the pole was assumed to be accurately reflected by the radius of the cylinder remote from the poles. He found values of $n$ varying from 0.6 to $2 \cdot 0$, ranging from the most pointed to the most blunt cell pole in the series. While the Cassinian equation and equation (15) are mathematically different, the predicted shapes are similar. Examples of fits to the Cassinian curves are also shown in Fig. 8 (dashed lines). The differences are significant and will be tested in future work.

\section{FUSIF ORM B ACTERIA}

As with the other bacterial shapes, several models within the general theory can be proposed for fusiform organisms. Two zonal models yielding poles with pointed ends are possible. One of these assumes that $P / T$ varies as the pole develops. Evidently, for a zonal 




Fig. 9. Fusiform cell shapes. The zonal growth model, as formulated by Koch et al. (1981 $a)$, was used to generate potential shapes for fusiform organisms. Each curve represents one-quarter of the cell profile. Two parameters are required by the model, $P / T$ and $\delta$. These are assumed to be constant throughout the cell cycle. The values of $P / T$ employed are shown on each curve. The different lines correspond to the following values of $\delta$ : continuous, $\delta=2 \cdot 1$; dashed, $\delta=2 \cdot 5$; dash-dotted, $\delta=3$.

model almost any shape of pole could be fitted, as long as $P / T$ can be taken as an arbitrary, variable quantity. The other type of zonal model is simpler in that it assumes that $P / T$ and $\delta$ are constant throughout the growth of the pole. The only modification of the theory applicable to $S$. faecium needed in this case is the change of $\delta$ to a value greater than 2 (see Fig. 9). Note that if $P / T$ is small, the poles are cone-shaped.

A model for diffuse growth is also possible. It requires, however, the assumption that $T$ varies, either with distance from the cell axis or with time of development of the pole. It is different from the zonal model mentioned above in that insertion of new wall material is possible all over the developing pole at any instant of time, but is similar in that most of the insertion of new material would occur near the splitting septum, even when the whole pole is capable of diffuse enlargement.

\section{VARIABLET MODELS OF GROWTH}

In contrast to fusiform bacteria, whose growth may be described by models assuming either constant or variable values for $T$, hyphal growth can be described only by variable $T$ models (Koch, 1982a). For Gram-negative rods and stalked bacteria, it is likely that $T$ varies during the cell cycle; the model for $E$. coli is developed elsewhere (Koch, 1982b).

\section{DIS CUSSION}

The general conclusion, based on the physics and mathematics of surface films, is that it is possible to explain the shapes of a variety of prokaryotes (and certain lower eukaryotes) using surface stress theory. The theory holds that tension in the wall in regions of growth determines the ultimate shape of the cell. For certain morphologies, more than one possible set of growth modes could produce the desired shapes, and future work will be needed to distinguish between the various possibilities and to test the general theory. In this paper, models for both zonal and diffuse wall growth have been presented to account for the growth pattern of (1) the nearly spherical cocci, (2) the sides of rod-shaped organisms, and (3) the poles of rod-shaped organisms. Since the hydrostatic pressure and the effective surface tension determine the shapes and sizes of organisms in this theory, there is no need to assume that there are special scaffolding devices created by the cell to define its own dimensions, as is the case with the replication of certain viruses (Casjens \& King, 1975). It is also unnecessary 


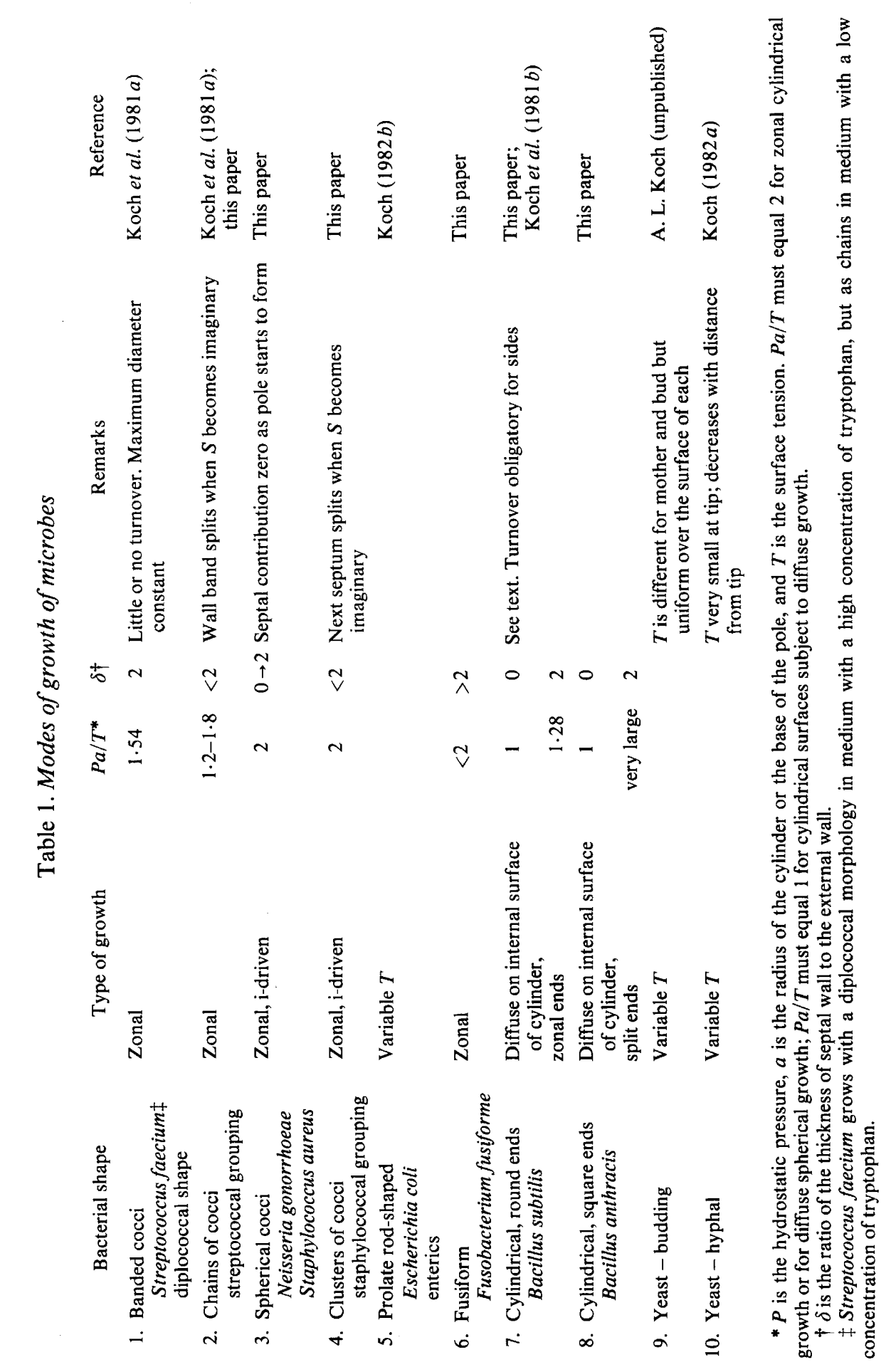


to assume that the structure and shape are inherently determined by the molecular properties of some macromolecule that serves as a building block which determines the supramolecular structure (Mendelson, 1976), such as certainly takes place in the formation of filamentous viruses via the polymerization of the coat protein(s) (Casjens \& King, 1975).

Although there are probably momentary increases that trigger the splitting of wall bands or the initiation of growth zones, the proposed models tacitly assume that the enlargement of wall is regulated in such a way that the pressure remains substantially constant throughout the cell cycle. The constant hydrostatic pressure could be achieved if the enzyme that hydrolyses selectively stressed peptidoglycan linkages is the rate-limiting step in wall enlargement and responds sensitively to the tension in those bonds. It was calculated earlier (Koch et al., 1981a) that the stress in the bonds would markedly lower the energy of activation and could lead to a large increase in the rate of hydrolysis. As long as the rate of glycan addition is not limiting, such a tension-responsive enzyme would lead to a constant pressure inside the cell. At the present time, these ideas are hypothetical, but reasonable. No assay has yet been devised to directly measure the kinetics of any enzyme acting on a substrate under controlled degrees of mechanical strain.

For microbes with different morphologies, different assumptions regarding the value of $T$ in different regions of the cell envelope are appropriate. Table 1 summarizes the special conditions needed for a number of prokaryote types. For many, $T$ appears to be constant, but Table 1 also includes Gram-negative rods and the yeast and mycelial growth phases of fungi, discussed in more detail elsewhere, for which $T$ does not appear to be constant. Although both zonal and diffuse models can generate poles and cylindrical side walls, we have chosen the more probable models for the various cell types. Thus, only a zonal model is proposed for the banded cocci which have diplococcal morphology with clearly discontinuous surfaces. A much more complex diffuse model with variable $T$ for this case has been omitted from consideration because the simple zonal model fits the shape of $S$. faecium so well. Similarly, only the zonal i-driven model is proposed for the spherical cocci largely because it explains more easily the formation of cell groups.

The picture for Gram-negative rods has recently been confused by the finding that the diameter changes systematically through the cell cycle (Trueba \& Woldringh, 1980). This greatly increases the possibilities and 13 models are considered (Koch, 1982 b) for wall growth in the Gram-negative enteric bacteria. However, Table 1 includes only a variable $T$ model, which we think is most probable.

There is an a priori reason to choose a diffuse model in preference to a zonal model for the cylindrical elongation of Gram-positive rods. The zonal model for diffuse elongation has the inherent difficulty that some secondary mechanism is needed to keep the cylinder from widening catastrophically, while such a special mechanism is not needed for the diffuse model. To make the point clear, consider a spherical soap bubble connected to a supply of constant pressure. If the pressure is greater than $2 T / r$, then the bubble will swell indefinitely; if it is less than this, it will shrink to nothing. This relationship also applies to zonal growth of a cylinder. However, a cylindrical structure, terminated by fixed radii, produced by diffuse growth at a fixed pressure is stable to fluctuation in that pressure, as long as the cylindrical region of growth does not become too long. Consequently, we suspect that truly cylindrical rods generally may have diffuse growth on their sides and probably not on their poles unless there are some special regulatory mechanisms in addition to those so far envisaged.

Probably the most significant conclusion of this paper is that it is theoretically possible for diffuse growth to lead to cylindrical elongation. This can only happen, however, when several conditions are met, i.e. $P$ is constant, $T$ is constant, the cell poles are rigid, and the elongating region is not longer than the circumference. The requirements of this type of growth are met by Gram-positive rods (see above and Koch et al., 1981 b). The inside-to-out growth of side walls is in strong contrast to the zonal poles which appear to turn over slowly or not at all, as expected by the theory. 
In principle, stress-bearing wall cannot turn over in the classical biochemical sense. A glycan unit in a stressed wall must have an extended conformation. Therefore, even if it was cleaved out and the remaining wall was strong enough to prevent rupture, there would be no way of introducing a new unit to replace the old. From the calculations presented previously (Koch et al., 1981 a) there is insufficient energy in the transpeptidation process both to form the new bond and to force it into an extended conformation. On the other hand, if a large glycan unit replaced a smaller unit removed at random, the process could be thermodynamically feasible, but the cell would necessarily become larger and more spherical. The models presented here and previously avoid these complications by linking the unit before cleavage occurs to transfer the stress.

For Gram-positive rods, there appear to be two observations contradictory to the inside-to-out growth model for cylinder elongation. One is the existence of mutants with little or no turnover that are still capable of normal growth. These cases need not be exceptions because the model only requires cleavage to transfer the stress to previously unstressed peptidoglycan residues, which are then forced into an extended conformation to allow growth. The fate of the old material no longer supporting stress is irrelevant, and in lytic-negative mutants may accumulate as peripheral aggregates or as feathery looking material. The other apparent exception is the thickened wall material resulting from wall formed during chloramphenicol treatment. Since turnover stops (Daneo-Moore \& Shockman, 1977), the material laid down during drug treatment may never be stressed until the drug is removed and both growth and turnover ensue. Such wall may never have supported the surface tension and its later disappearance is irrelevant to the growth of stress-bearing wall.

It is hoped that the physical theory presented here will help to define the complex biological processes leading to the range of microbial shapes. It may be that ultra-histochemical assay or ultra-high resolution autoradiography of pulse-labelled cells will be able to demonstrate the location of wall synthesis unambiguously. In cases of zonal growth, the cell must have regulatory mechanisms to initiate a growth zone and have ways to restrict the growing region to keep it narrow. The latter might be done by the continuous secretion of enzymes with short half-lives, only at the growth zone. Alternatively, enzymes may be formed in a burst only at the time of initiation of a growth zone, and only remain functional during the life of the growth zone. These two possibilities would give rise to linear growth for the cylindrical walls if the enzymes were limiting, but not if the enzymes are in excess and respond to tension in the wall.

The most intriguing aspect of this work is the possibility of a generalization that may have phylogenetic implications. It may be that all the banded cocci only grow zonally and can form only linear chains. They may adjust their growth rate to ambient conditions by triggering the frequency of wall band splitting in response to faster protoplasm accumulation. This mode may be available only to Gram-positive organisms. It may be that the unbanded spherical cocci, whether Gram-positive or Gram-negative, cannot form linear chains. Instead, they create de novo sites of septum formation in some other plane and adjust their growth rate by the frequency of such initiation. Growth may be diffuse in all bacteria with unbanded diplococcal morphology. It may be that rod-shaped organisms consist of two fundamentally different classes - the truly cylindrical rods and rods that more closely approach the shape of eccentric prolate ellipsoids. The former may always have juncture sites between the cylinder and pole that are formed in quantitatively different ways, and the latter not.

The segregation of DNA to daughter cells can be explained by the replicon model of Jacob et al. (1963), if amended to assume that the origin of DNA replication binds through the cytoplasmic membrane to the wall; but this model cannot account for segregation if wall growth occurs outside a central region limited by the attachment of the replication origins. A development of the original replicon model may overcome this difficulty and explain some newer data concerning $B$. subtilis (Koch et al., 1981 b). The new model postulates that there is a specialized site (juncture site) at the junction of the cylindrical region and the caps to which the origins and termini of the chromosomes bind. We have suggested that these may be 
located at a point within the wall band identified by Burdett \& Higgins (1978). We assume that binding to this juncture site accounts for the special fraction of DNA found bound to the wall (Doyle et al., 1980). Equivalent models must be made and tested for all bacteria with diffuse wall growth.

John Chadam of the Indiana University Mathematics Department casually wrote out the treatment for the slope of an arbitrary unduloid. This served as the basis for the computer program employed. We also thank David Fan for early ideas about the structure of the Gram-positive wall. Experimental work leading to these theoretical considerations was supported by NSF PCM 79-11241 (A.L.K.), NIH AI 10971 (M.L.H.) and NSF PCM $78-08903$ (R.J.D.).

\section{REFERENCES}

Anderson, A. J., Green, R. S., Sturman, A. J. \& ARCHIBALD, A. R. (1978). Cell wall assembly in Bacillus subtilis: location of wall material incorporated during pulse release of phosphate limitation; its accessibility to bacteriophages and concanavalin $A$, and its susceptibility to turnover. Journal of Bacteriology 136, 886-899.

ARChiBALD, A. R. (1976). Cell wall assembly in Bacillus subtilis: development of bacteriophage binding properties as a result of the pulsed incorporation of teichoic acid. Journal of Bacteriology 127, 956-960.

Archibald, A. R. \& Coapes, H. E. (1976). Bacteriophage SP50 as a marker for cell wall growth in Bacillus subtilis. Journal of Bacteriology 125, 1195-1206.

BurdetT, I. D. J. \& Higgins, M. L. (1978). Studies of pole assembly in Bacillus subtilis by computer reconstruction of septal growth zones seen in central, longitudinal, thin sections of cells. Journal of Bacteriology 133, 959-971.

CASJENS, S. \& KING, J. (1975). Virus assembly. Annual Review of Biochemistry 44, 555-611.

Daneo-Moore, L. \& Shockman, G. D. (1977). The bacterial cell surface in growth and division. In The Synthesis, Assembly and Turnover of Cell Surface Components, pp. 597-715. Edited by G. Poste \& G. L. Nicolson. Amsterdam: Elsevier/North Holland.

Delaunay, C. (1841). Sur la surface de révolution dont la courbure moyenne est constant. Journal de mathématiques pures et appliquées 6, 309-315.

Doyle, R. J., Streips, U. N., Imada, S., Fan, V. S. C. \& Brown, W. C. (1980). Genetic transformation with cell wall-associated deoxyribonucleic acid in Bacillus subtilis. Journal of Bacteriology 144, 957-966.

Doyle, R. J., Mobley, H. L. T., Streips, U. N. \& JoLlifFE, L. K. (1981). Restricted turnover of the walls of Bacillus subtilis. Current Microbiology 5, 19-22.

FAN, D. P., Beckman, B. E. \& Gardner-Eckstrom, H. L. (1975). Mode of cell wall synthesis in gram-positive bacteria. Journal of Bacteriology 123 , 1157-1162.

Giesbrecht, P., Wecke, J. \& Reinicke, B. (1976). On the morphogenesis of the cell wall of staphylococci. International Review of Cytology 44, 225318.

ISENBERG, C. (1978). The Science of Soap Films and Soap Bubbles. Bridgewater: Advanced Educational Toys, Ltd.
Jacos, F., Brenner, S. \& Cuzin, F. (1963). On the regulation of DNA replication in bacteria. Cold Spring Harbor Symposia on Quantitative Biology 28, 329-347.

KocH, A. L. $(1982 a)$. The shape of the hyphal tips of fungi. Journal of General Microbiology 128, 947951.

KoCH, A. L. $(1982 b)$. On the growth and form of Escherichia coli. Journal of General Microbiology 128 (in the Press).

Koch, A. L., Higgins, M. L. \& Doyle, R. J. (1981a). Surface tension-like forces determine bacterial shapes: Streptococcus faecium. Journal of General Microbiology 123, 151-161.

Koch, A. L., Mobley, H. L. T., Doyle, R. J. \& Streips, U. N. $(1981 b)$. The coupling of wall growth and chromosome replication in Gram positive rods. FEMS Microbiology Letters 12, 201-208.

LAPLACE, P. S. (1806). Mécanique Céleste. Paris: Imprimeur Imperiale. (Supplement to the tenth book.)

Maxwell, J. C. (1849). On the theory of rolling curves. Transactions of the Royal Society of Edinburgh 16, 519-540.

MAXWELL, J. C. (1927). Capillary action. Reprinted in The Scientific Papers of James Clerk Maxwell, vol. 2, pp. 541-591. Paris: Libraire Scientifique, J. Hermann.

Mendelson, N. H. (1976). Helical growth of Bacillus subtilis: a new model of cell growth. Proceedings of the National Academy of Sciences of the United States of America 73, 1740-1744.

Pooley, H. M. (1976a). Turnover and spreading of old wall during surface growth of Bacillus subtilis. Journal of Bacteriology 125, 1127-1138.

Pooley, H. M. (1976b). Layered distribution according to age within the cell wall of Bacillus subtilis. Journal of Bacteriology 125, 1139-1147.

Sturman, A. J. \& Archibald, A. R. (1978). Conservation of phage receptor material at the polar caps of Bacillus subtilis W23. FEMS Microbiology Letters 4, 255-259.

THOMPSON, D'ARCY W. (1942). On Growth and Form, 2nd edn, pp. 351-384. Cambridge: Cambridge University Press.

Trueba, F. J. \& Woldringh, C. L. (1980). Changes in cell diameter during the division cycle of Escherichia coli. Journal of Bacteriology 142, 869-878.

Young, T. (1805). Cohesion of fluids. Philosophical Transactions of the Royal Society 95, 65-87. 